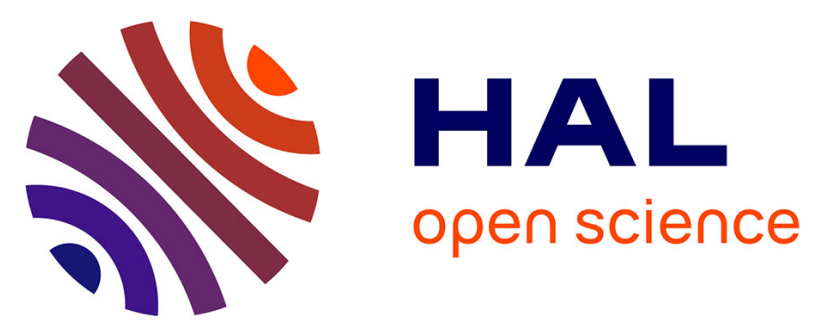

\title{
Détermination de la masse d'eau et des propriétés optiques d'une feuille à partir de modifications de son bilan énergétique. I. - Bases théoriques de la méthode et technique de mesure
}

Galileo Adeli Buriol, Fernando Santibañez, Yvette Menoux, Sané de

Parcevaux, Jean-Michel Bertolini

\section{To cite this version:}

Galileo Adeli Buriol, Fernando Santibañez, Yvette Menoux, Sané de Parcevaux, Jean-Michel Bertolini. Détermination de la masse d'eau et des propriétés optiques d'une feuille à partir de modifications de son bilan énergétique. I. - Bases théoriques de la méthode et technique de mesure. Agronomie, 1984, 4 (6), pp.493-500. hal-00884664

\section{HAL Id: hal-00884664 https://hal.science/hal-00884664}

Submitted on 1 Jan 1984

HAL is a multi-disciplinary open access archive for the deposit and dissemination of scientific research documents, whether they are published or not. The documents may come from teaching and research institutions in France or abroad, or from public or private research centers.
L'archive ouverte pluridisciplinaire HAL, est destinée au dépôt et à la diffusion de documents scientifiques de niveau recherche, publiés ou non, émanant des établissements d'enseignement et de recherche français ou étrangers, des laboratoires publics ou privés. 


\section{Détermination de la masse d'eau et des propriétés optiques d'une feuille à partir de modifications de son bilan énergétique. I. - Bases théoriques de la méthode et technique de mesure.}

Galileo Adeli BURIOL, Fernando SANTIBAÑEZ $(*)$, Yvette MENOUX $\left({ }^{* *}\right)$, Sané de PARCEVAUX $\left({ }^{* *}\right) \&$ Jean-Michel BERTOLINI (**)

Centro de Ciencias rurais, Universidad federal de Santa Maria, BR Santa Maria, Brésil.

(*) Facultad de Ciencias agrarias y veterinarias, Universidad de Chile, CL Santiago, Chili.

(**) I.N.R.A., Station de Bioclimatologie, Route de Saint-Cyr, F 78000 Versailles

A partir d'une étude de la cinétique de la température de la feuille après modification de son bilan d'énergie, nous proposons une méthode non destructive qui permet de déterminer, d'une part, la masse d'eau surfacique des feuilles, d'autre part, certaines de leurs propriétés optiques. La méthode utilise un dispositif d'apport d'énergie radiative additionnel $(\Delta \phi)$ et un dispositif de mesure de l'écart thermique $(\Delta T)$ entre 2 points d'une même feuille, distants de $2 \mathrm{~cm}$ environ. Le premier permet l'obtention d'un spot lumineux relativement homogène et d'une densité de flux moyenne de $210 \mathrm{~W} . \mathrm{m}^{-2}$; le second se compose d'un capteur thermique différentiel (thermocouples) et d'un système automatique d'enregistrement de la pente à l'origine de la cinétique thermique $(\Delta \mathrm{T} / \Delta \mathrm{t})$.

Grâce à son « caractère différentiel » cette méthode peut être appliquée aussi bien en conditions de laboratoire qu'en conditions naturelles.

Mots clés additionnels : Capteur thermique différentiel, cinétique thermique, écart thermique, rayonnement additionnel, thermocouple.

Determination of the water content and optical properties of a leaf from changes in its energy balance.

\section{I. - Theoretical bases of the method and measuring technique.}

Leaf water content can be determined using a non destructive method based upon the observation of leaf temperature kinetics following changes in leaf energy balance. As a result of this method, the leaf surface caloric capacity can be evaluated, and hence the leaf water content. Leaf optical properties can also be investigated.

For measuring the leaf surface caloric capacity this method uses a device providing additional radiative energy $(\Delta \phi)$ and a device for measuring the thermal difference $(\Delta T)$ between 2 points of the same leaf which are approx. $2 \mathrm{~cm}$ apart. A relatively homogeneous light spot of mean flux density $210 \mathrm{~W} . \mathrm{m}^{-2}$ can be obtained with the first device ; the second device includes a differential thermal sensor (thermocouples) and an automatic system for recording the slope initiating the thermokinetics $(\Delta T / \Delta t)$.

Due to its "differential character", this method may be applied both to field and laboratory experiments.

Additional key words : Differential thermal sensor, thermokinetics, thermal difference, additional radiation, thermocouple.

\section{INTRODUCTION}

Les méthodes classiques de quantification de l'état hydrique de la feuille ont l'inconvénient d'introduire des modifications irréversibles au cours de l'étude, notamment par destruction. Au cours de ces dernières années, des efforts ont été faits dans la recherche de méthodes «non destructives » et ne perturbant pas l'environnement végétal. Elles permettent ainsi de suivre les variations de la masse d'eau d'un même organe pendant un temps qui peut être plus ou moins long (un jour, plusieurs jours, ou le cycle de la feuille). 
TABLEAU I

Signification des symboles.

Meaning of symbols.

\begin{tabular}{|c|c|}
\hline $\mathrm{C}$ & $\begin{array}{l}\text { Chaleur massique moyenne de la feuille (Mean leaf } \\
\text { specific heat) }\left(\mathrm{J} . \mathrm{kg}^{-1} \cdot \mathrm{K}^{-1}\right)\end{array}$ \\
\hline $\mathrm{Ce}$ & $\begin{array}{l}\text { Chaleur massique de l'eau (Water specific heat) } \\
\left(4180 \mathrm{~J} \cdot \mathrm{kg}^{-1} \cdot \mathrm{K}^{-1}\right)\end{array}$ \\
\hline $\mathrm{Cp}$ & $\begin{array}{l}\text { Chaleur massique de l'air (Air specific heat) } \\
\left(1010 \mathrm{~J} \cdot \mathrm{kg}^{-1} \cdot \mathrm{K}^{-1}\right)\end{array}$ \\
\hline $\mathrm{Cs}$ & $\begin{array}{l}\text { Chaleur massique de la matière sèche (Dry matter } \\
\text { specific heat) }\left(\mathrm{J} \cdot \mathrm{kg}^{-1} \cdot \mathrm{K}^{-1}\right)\end{array}$ \\
\hline M & $\begin{array}{l}\text { Masse surfacique de la feuille (Surface mass of the } \\
\text { leaf) }\left(\mathrm{kg} \cdot \mathrm{m}^{-2}\right)\end{array}$ \\
\hline MC & $\begin{array}{l}\text { Capacité calorifique foliaire surfacique (Leaf surface } \\
\text { caloric capacity) }\left(\mathrm{J} \cdot \mathrm{m}^{-2} \cdot \mathrm{K}^{-1}\right)\end{array}$ \\
\hline $\mathrm{Me}$ & $\begin{array}{l}\text { Masse d'eau foliaire surfacique (Leaf surface water } \\
\text { content) }\left(\mathrm{kg} \cdot \mathrm{m}^{-2}\right)\end{array}$ \\
\hline Ms & $\begin{array}{l}\text { Masse surfacique de la matière sèche (Surface } \\
\text { mass of the dry matter) }\left(\mathrm{kg} \cdot \mathrm{m}^{-2}\right)\end{array}$ \\
\hline $\mathbf{P}^{\prime}$ & $\begin{array}{l}\text { Pente moyenne de la courbe représentant les ten- } \\
\text { sions maximales de vapeur d'eau en fonction des } \\
\text { températures (Mean slope of maximal vapor pressure } \\
\text { curve versus temperature) (Pa } . \mathrm{K}^{-1} \text { ) }\end{array}$ \\
\hline $\mathrm{r}_{\mathrm{a}}$ & 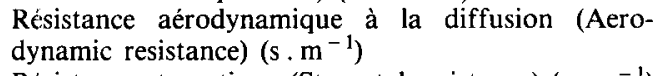 \\
\hline $\mathbf{r}_{\mathrm{s}}$ & Résistance stomatique (Stomatal resistance) $\left(\mathrm{s} \mathrm{.} \mathrm{m}^{-1}\right)$ \\
\hline $\mathbf{T}$ & Température de la feuille (Leaf temperature) (K) \\
\hline $\mathrm{Ta}$ & Température de l'air (Air temperature) $(\mathrm{K})$ \\
\hline To & $\begin{array}{l}\text { Température initiale d'équilibre (Initial stable tem- } \\
\text { perature of the leaf) (K) }\end{array}$ \\
\hline $\operatorname{Tr}$ & $\begin{array}{l}\text { Température du point de rosée en valeur absolue } \\
\text { (Dew point temperature) }(\mathrm{K})\end{array}$ \\
\hline$\gamma$ & $\begin{array}{l}\text { Constante psychrométrique (Psychrometric constant) } \\
\left(66 \mathrm{PaK}^{-1}\right)\end{array}$ \\
\hline$\Delta \mathbf{T}=\mathrm{T}-\mathrm{To}$ & $\begin{array}{l}\text { Différence de température entre le point de la feuille } \\
\text { situé sous le spot lumineux et un point servant de } \\
\text { référence (Difference of temperature between the } \\
\text { leafspot under the spotlight and a nearby reference } \\
\text { point) (K) }\end{array}$ \\
\hline$\Delta \mathrm{T} / \Delta \mathrm{t}$ & $\begin{array}{l}\text { Vitesse initiale de variation de la température } \\
\text { foliaire en présence de rayonnement additionnel } \\
\text { (Initial rate of leaf temperature variation under } \\
\text { additional radiation) }\left(\mathrm{K} \cdot \mathrm{s}^{-1}\right)\end{array}$ \\
\hline$\Delta \mathrm{t}$ & Intervalle de temps (Time range) (s) \\
\hline$\Delta \phi_{\mathrm{a}}$ & $\begin{array}{l}\text { Densité du flux de rayonnement additionnel absorbé } \\
\text { (Absorbed additional radiation flux) }\left(\mathrm{W} . \mathrm{m}^{-2}\right)\end{array}$ \\
\hline$\epsilon$ & $\begin{array}{l}\text { Facteur d'émissivité propre à la feuille (Leaf } \\
\text { emissivity) }(\sim 0,95)\end{array}$ \\
\hline$\phi \mathrm{a}$ & $\begin{array}{l}\text { Densité de flux de rayonnement absorbé (Absorbed } \\
\text { radiation flux) (W. m-2) }\end{array}$ \\
\hline$\phi \mathrm{e}$ & $\begin{array}{l}\text { Densité de flux de rayonnement émis (Emitted radia- } \\
\left.\text { tion flux) (W. } \mathrm{m}^{-2}\right)\end{array}$ \\
\hline$\phi \mathrm{H}$ & $\begin{array}{l}\text { Densité de flux des échanges convectifs (Convective } \\
\text { exchanges flux) (W. } \mathrm{m}^{-2} \text { ) }\end{array}$ \\
\hline$\phi \mathrm{L}$ & $\begin{array}{l}\text { Densité de flux de chaleur latente (Latent heat } \\
\text { flux) }\left(\mathrm{W} . \mathrm{m}^{-2}\right)\end{array}$ \\
\hline$\varrho$ & Masse volumique de l'air \\
\hline & $\begin{array}{l}\text { 1stante de Stefan-Bol } \\
\text { stant) }\left(5,673 \cdot 10^{-8}\right.\end{array}$ \\
\hline
\end{tabular}

- soit la variation de la conductivité électrique des tissus (BOX \& LEMON, 1958 ; NAMKEN \& LEMON, 1960 ; KREEB, 1966 ; KREEB \& ABDELALL, 1973) ;

- soit la variation de la réflexion de radiation incidente par la surface foliaire (THOMAS et al., 1971) ;

- soit la mesure de la constante diélectrique (GEZALYAN, 1966, 1968).

Pour toutes ces méthodes l'étalonnage est souvent le point délicat, soit parce que la méthode n'est pas sélective pour l'eau (rayons bêta et gamma), soit en raison de l'hétérogénéité de la structure du couvert végétal ou de la feuille (ondes électromagnétiques et conductivité électrique), ou d'une mise au point technique particulièrement difficile (constante diélectrique).

Le but de cette étude est de présenter le principe théorique d'une nouvelle méthode et la mise au point d'une technique non destructive appropriée pour la détermination in situ de la masse d'eau foliaire. Cette méthode permet également d'étudier les variations des propriétés optiques de la feuille en fonction de sa masse d'eau (SANTIBAÑEZ, 1974 ; BURIOL et al., 1984). Elle est basée sur l'étude de la cinétique de la température de la feuille après modification de son bilan énergétique. Les travaux expérimentaux ont été réalisés à la Station de Bioclimatologie de l'I.N.R.A., à Versailles.

\section{THÉORIE : ÉTUDE DES VARIATIONS DU BILAN D'ÉNERGIE D'UNE SURFACE DE} FAIBLE ÉPAISSEUR (FEUILLE D'UNE PLANTE)

Des modifications artificielles du bilan énergétique foliaire sont classiquement utilisées pour déterminer les résistances à la diffusion (IMPENS, 1966 ; HUNT \& IMPENS, 1968 ; LINACRE, 1972 ; PARCEVAUX, 1972a, $b$ ). PARCEVAUX \& PERRIER (1973) ont envisagé l'introduction de telles modifications pour déterminer la masse d'eau de la feuille.

\section{A. Bilan d'énergie d'une feuille}

Le bilan global d'énergie d'une feuille peut s'écrire en négligeant le flux utilisé dans le processus photochimique :

$$
\Phi \mathrm{a}+\Phi \mathrm{e}+\Phi \mathrm{H}+\Phi \mathrm{L}-\mathrm{MC} \frac{\partial \mathrm{T}}{\partial \mathrm{t}}=0
$$

Par convention, le signe $(+)$ signifie un gain d'énergie (cf. tabl. 1).

Le produit MC représente la capacité calorifique foliaire rapportée à l'unité de surface. Il correspond aussi à « l'inertie thermique » de la feuille. C'est le paramètre important pour la théorie et l'application de la méthode préconisée.

\section{B. Etude d'une variation provoquée du bilan énergé- tique de la feuille}

A l'état d'équilibre, le bilan d'énergie de la feuille peut être reécrit en fonction des paramètres intervenant sur la valeur de chaque terme (PARCEVAUX \& PERRIER, 1973). Si To représente la température à 
l'état d'équilibre, on a :

$$
\Phi \mathrm{a}-2 \varepsilon \sigma \mathrm{T}_{0}^{4}-2 \rho \operatorname{Cp}\left(\frac{\mathrm{To}-\mathrm{Ta}}{\mathrm{r}_{\mathrm{a}}}+\frac{\mathrm{P}^{\prime}}{\gamma} \cdot \frac{\mathrm{To}-\mathrm{Tr}}{\mathrm{r}_{\mathrm{a}}+\mathrm{r}_{\mathrm{s}}}\right)=0
$$

le dernier terme de l'équation (1) : $\mathrm{MC} \delta \mathrm{T} / \delta \mathrm{t}$ étant nul.

L'introduction d'un flux radiatif additionnel absorbé $\Delta \Phi$ a provoque un réajustement du bilan éner- gétique de la feuille au cours duquel la température de surface $T$ évolue selon l'équation différentielle suivante :

$$
\Delta \Phi \mathrm{a}+\Phi \mathrm{a}-2 \varepsilon \sigma \mathrm{T}^{4}-2 \rho \mathrm{Cp}\left(\frac{\mathrm{T}-\mathrm{Ta}}{\mathrm{r}_{\mathrm{a}}}+\frac{\mathrm{P}^{\prime}}{\gamma} \cdot \frac{\mathrm{T}-\mathrm{Tr}}{\mathrm{r}_{\mathrm{a}}+\mathrm{r}_{\mathrm{s}}}\right)-\mathrm{MC} \frac{\partial \mathrm{T}}{\partial \mathrm{t}}=0
$$

En posant $c=4+\frac{6(\mathrm{~T}-\mathrm{To})}{\mathrm{To}_{\mathrm{O}}} \# 4,1, \quad$ on obtient en soustrayant $(2)$ de $(3)$ :

$$
\Delta \Phi \mathrm{a}-2(\mathrm{~T}-\mathrm{To})\left(\operatorname{c\varepsilon } \sigma \mathrm{T}^{3}+\rho \mathrm{Cp}\left(\frac{1}{\mathrm{r}_{\mathrm{a}}}+\frac{\mathrm{P}^{\prime}}{\gamma\left(\mathrm{r}_{\mathrm{a}}+\mathrm{r}_{\mathrm{s}}\right)}\right)\right)-\mathrm{MC} \frac{\hat{c \mathrm{~T}}}{\partial \mathrm{t}}=0 .
$$

Immédiatement après l'application de $\Delta \Phi a$, ( $\mathrm{T}-\mathrm{To}$ ) a une valeur négligeable, d'où :

$$
\Delta \Phi_{\mathrm{a}}=\mathrm{MC}\left(\frac{\partial \mathrm{T}}{\partial \mathrm{t}}\right)_{\mathrm{t}=0}
$$

La figure 1 montre les différentes phases de la cinétique thermique foliaire observée consécutivement à une variation de l'énergie radiative absorbée.

La pente à l'origine de la cinétique de réchauffement conduit à la connaissance de la capacité calorifique surfacique MC de la feuille si le flux de rayonnement additionnel absorbé est connu. Inversement, si $\mathrm{MC}$ est connue, le flux absorbé $\Delta \Phi$ a peut être déterminé.
Les études du bilan énergétique ont été effectuées pour la plupart en conditions climatiques contrôlées où un régime permanent peut être obtenu. En conditions naturelles où la feuille est soumise à des variations des paramètres microclimatiques et physiologiques, une mesure différentielle s'impose. Cela revient à mesurer l'écart thermique entre 2 points d'une feuille, dont l'un seulement reçoit le rayonnement additionnel. Pour obtenir une compensation parfaite, il est essentiel que l'état physique et biologique des 2 points soit identique. Une distance de séparation suffisamment faible $(2 \mathrm{~cm}$ alors que la surface éclairée est de $1 \mathrm{~cm}^{2}$ environ) assure cette condition, qui est confirmée par l'obtention d'un écart nul en l'absence du rayonnement additionnel.

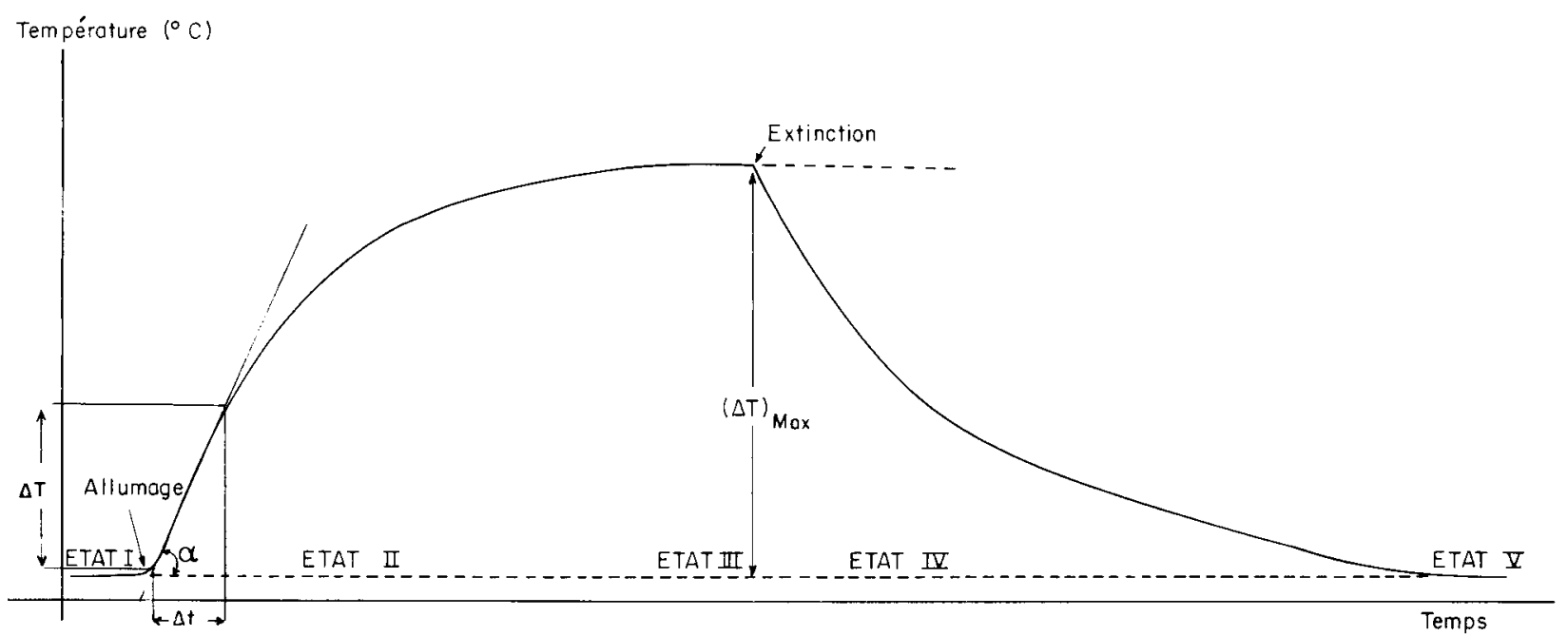

Figure 1

Différentes phases de l'évolution de la température foliaire en réponse à une variation du rayonnement absorbé. I. Equilibre initial à la température To (équation 2). II. Cinétique d'échauffement en présence du flux radiatif additionnel absorbé $\Delta \phi a ;$ la pente à l'origine $\alpha=\Delta T / \Delta t$ est égale à $\Delta \phi a / M C$. III. Nouvel état d'équilibre. $I V$. Cinétique de refroidissement après suppression du rayonnement additionnel. V. Retour à l'équilibre initial To.
Different stages of the kinetics of leaf temperature when the radiation absorbed is changed. I. First equilibrium at temperature To (equation 2). II. Increasing temperature due to additional radiative flux absorbed $\Delta \phi a$; initial slope $\alpha=\Delta T / \Delta t$ is equal to $\Delta \phi a / M C$. III. New state of equilibrium. IV. Temperature decreasing after removal of additionnal radiation. $V$. Return to the initial equilibrium To. 
$\Delta \mathrm{T}$ étant l'écart thermique entre ces 2 points, de (5) on tire :

$$
\Delta \Phi \mathrm{a}=\operatorname{MC}\left(\frac{\partial(\Delta \mathrm{T})}{\hat{\partial t}}\right)_{\mathrm{t}=0}
$$

\section{Calcul de la masse d'eau foliaire}

La capacité calorifique surfacique de la feuille peut se décomposer en 2 éléments :

$$
\mathrm{Mc}=\mathrm{Me} . \mathrm{Ce}+\mathrm{Ms} . \mathrm{Cs} .
$$

Dans cette équation, Me est la valeur recherchée ; $\mathrm{Ce}$ a une valeur définie soit $1 \mathrm{cal} \cdot \mathrm{g}^{-1} \cdot \mathrm{K}^{-1}$ ou $4180 \mathrm{~J} \cdot \mathrm{kg}^{-1} \cdot \mathrm{K}^{-1}$ et Ms représente de façon générale, 3 à 10 p. 100 de Me. Une valeur de 5 p. 100 est très proche de la réalité et peut être utilisée dans les cas où l'on ne connaît pas le contenu exact en matière sèche.

On peut prendre pour $C$ s une valeur moyenne de $0,3 \mathrm{cal} \cdot \mathrm{g}^{-1} \cdot \mathrm{K}^{-1}$ ou $1250 \mathrm{~J} \cdot \mathrm{kg}^{-1} \cdot \mathrm{K}^{-1}$, sachant qu'une variation de \pm 25 p. 100 de ce paramètre entraînera une erreur inférieure à \pm 2 p. 100 sur l'estimation de la masse calorifique surfacique de la feuille (PARCEVAUX, 1972a).

La relation entre la masse calorifique $\mathrm{MC}$ et la masse en eau surfacique Me peut alors être écrite :

$$
\begin{aligned}
& \mathrm{Me}=(\mathrm{MC}-0,3 \mathrm{Ms}) \sim 0,95 \mathrm{MC} . \\
& \text { III. CHOIX DES MATÉRIELS ET } \\
& \text { MONTAGE DE L'APPAREIL }
\end{aligned}
$$

\section{CHOIX DES MATÉRIELS ET MONTAGE DE L'APPAREIL}

La mise en œuvre de cette méthode exige la solution de quelques problèmes techniques dont l'étude de l'intensité et de la composition spectrale du rayonnement additionnel nécessaire à la modification du bilan énergétique et la mise au point d'un système permettant la détermination rapide et précise de l'écart thermique $(\Delta \mathrm{T})$ entre 2 points d'une feuille.

\section{A. Apport d'énergie}

Il est important que la perturbation introduite par l'apport énergétique additionnel sur les conditions physiologiques de la feuille soit négligeable et que la densité du flux additionnel absorbée par la feuille soit constante. En effet, le coefficient d'absorption doit rester indépendant de la masse d'eau, au moins dans la gamme des variations écologiques de ce paramètre. La $2^{\mathrm{e}}$ condition élimine pratiquement la possibilité d'utiliser le soleil comme source d'énergie. Un spot infrarouge est physiologiquement inactif en ce qui concerne l'ouverture des stomates et la photosynthèse, mais la littérature (BILLINGS \& MORRIS, 1951 ; KNIPLING, 1970 ; SINCLAIR et al., 1971 ; SANTIBAÑEZ, 1974) indique qu'il existe une étroite dépendance entre la teneur en eau de la feuille et le coefficient d'absorption dans l'infrarouge.

Ces faits nous ont amenés à utiliser un spot de lumière visible dont l'absorption varie d'une façon négligeable dans une large gamme de variations de la masse d'eau surfacique de la feuille. Pour obtenir l'écart minimal de 2 à $3{ }^{\circ} \mathrm{C}$ nécessaire à la détermination de la pente à l'origine de la cinétique thermique foliaire avec précision, une densité de flux de $210 \mathrm{~W} . \mathrm{m}^{-2}$ pendant $10 \mathrm{~s}$ suffit.
Un dispositif d'éclairage provenant d'un projecteur de diapositives (avec une lampe quartziode de $50 \mathrm{~W}$ ) a été utilisé. Un diaphragme, 2 lentilles additionnelles, un filtre anticalorique et un filtre bleu (LEITZ, BG 38, transmission entre 310 et $710 \mathrm{~nm}$ ) ont permis l'obtention d'un spot lumineux focalisé (fig. 2).

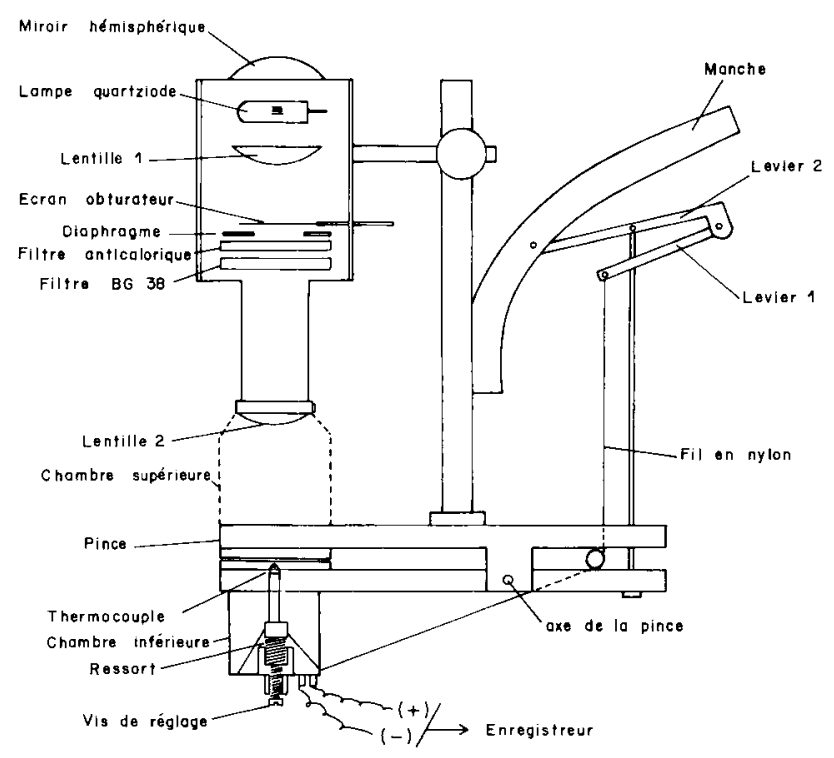

Figure 2

Montage utilisé pour la détermination de la teneur en eau en conditions naturelles.

Set-up used for determining leaf water mass in natural conditions.

\section{B. Détermination de la cinétique thermique}

Le dispositif de mesure de la cinétique thermique $(\Delta \mathrm{T} / \Delta \mathrm{t})$ de la feuille se compose de 2 parties : un capteur thermique différentiel et un système de détermination de la pente à l'origine de la cinétique thermique.

\section{Capteur thermique différentiel}

La température foliaire est un paramètre difficilement mesurable (PERRIER, 1971 ; PERRIER \& ITIER, 1975), ce qui implique de prendre en compte les impératifs suivants, pour la précision de l'ordre de $1 / 10^{\circ} \mathrm{C}$ requise :

- réduire au minimum toute perturbation de l'environnement et du matériel végétal lui-même,

- intégrer la mesure de température sur une surface représentative ( 1 ou plusieurs $\mathrm{cm}^{2}$ ) et, en tout cas, peu différente de la surface éclairée,

- avoir une inertie thermique et un temps de réponse des instruments inférieurs à ceux de la feuille.

Cela conduit à utiliser comme capteur soit un radiomètre infrarouge soit un thermocouple.

La radiométrie infrarouge (PERRIER, 1970) permet d'obtenir la température moyenne d'une aire importante sur la feuille (un ou plusieurs $\mathrm{cm}^{2}$ ). Les thermocouples permettent une mesure différentielle mais ponctuelle (DURAND, 1970). Un dispositif théoriquement idéal serait un radiomètre infrarouge différentiel. Mais il n'existe pas, jusqu'à présent, de capteurs différentiels par radiométrie infrarouge. Ainsi, malgré 
certains inconvénients, les thermocouples demeurent İes meilleurs capteurs utilisables actuellement pour la détermination d'un écart de température entre 2 points d'une feuille.

Les meilleurs résultats ont été obtenus en utilisant un thermocouple fin $(25 \mu)$ en forme de boucle soutenue par une petite boule en polystyrène expansé et appuyée avec une légère pression sur la feuille de façon à ce que le fil touche la surface de la feuille sur une longueur de 1 ou $2 \mathrm{~mm}$ de chaque côté de la soudure (fig. 3). Cette géométrie est essentielle pour réduire les échanges de chaleur avec l'air. Etant donné de plus la faible capacité thermique du fil du thermocouple devant celle de la feuille, la soudure prend rapidement la température de la feuille sans pratiquement la perturber.

Pour déterminer l'influence du polystyrène sur la mesure de la température de la feuille, un jeu de 2 thermocouples sans polystyrène a été fixé sur la face supérieure de la feuille, permettant ainsi d'enregistrer tout écart thermique entre les points 1 et 2 (fig. 3). La perturbation au point 1 introduite au moment où la feuille est touchée par sa face inférieure avec le thermocouple muni d'un support en polystyrène est de l'ordre de $1 / 10$ ou $2 / 10$ de degré et ne dure que quelques $1 / 10$ de secondes. Ces valeurs ont été obtenues en travaillant sur des feuilles de tournesol, de 0,25 à $0,30 \mathrm{~mm}$ d'épaisseur et peuvent être généralement considérées comme négligeables (SANTIBAÑEZ, 1974). Un dispositif analogue, mais en araldite, donc de propriétés thermiques beaucoup moins favorables, a été utilisé mais non testé par SCHULzE (1970).

Pour assurer une mesure différentielle, les 2 soudures d'un même thermocouple sont montées sur 2 supports distants de $2 \mathrm{~cm}$ environ. Les supports coudés tournent autour d'un axe de façon à former un système à bascule. Un contrepoids permet de régler la pression d'appui des thermocouples sur la feuille (fig. 4a). Pour le travail au champ, le système à bascule a été remplacé par un système à ressorts (fig. $4 b$ ). Dans ce dernier cas, la pression d'appui est réglée par une vis.

Les thermocouples et leurs supports sont fixés à l'intérieur d'une petite chambre munie d'une pince permettant la fixation correcte de l'ensemble sur la feuille. Bien qu'elle introduise une certaine perturba-

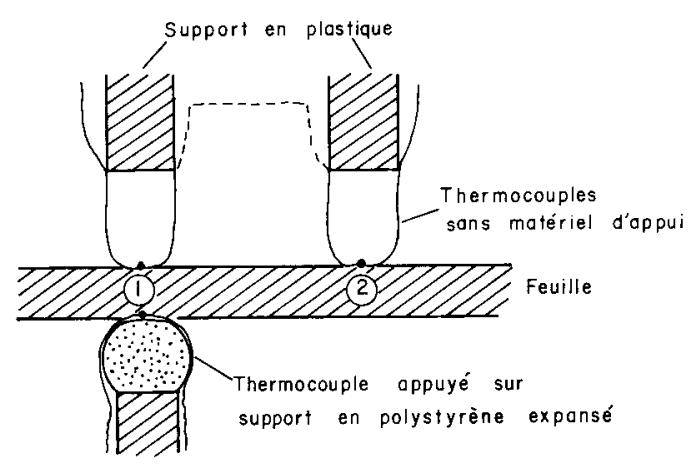

Figure 3

Montage des thermocouples pour étudier l'effet perturbateur du matériel d'appui.

Fitting of thermocouples for analysing the disturbing effect of the supporting material.

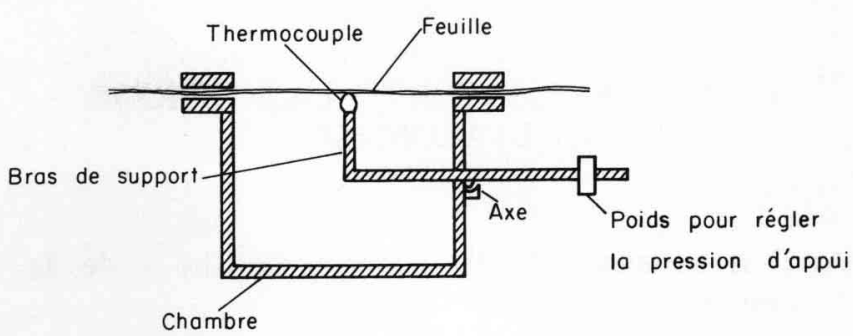

a. Système à bascule

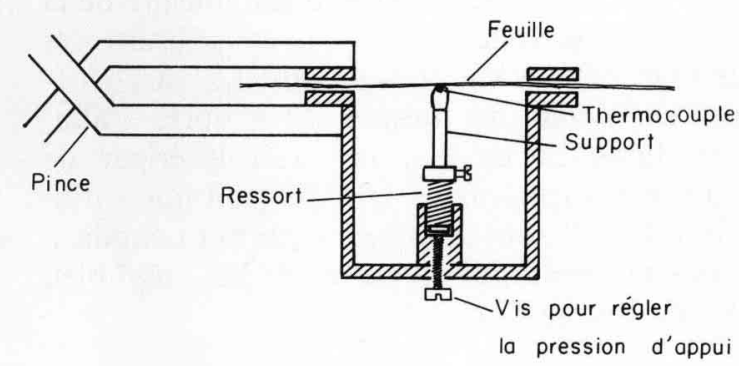

b. Système à ressorts

Figure 4

Détail des chambres de mesure de la température foliaire.

Systems used for determining leaf temperature.

tion microclimatique au niveau de la feuille, la chambre constitue un dispositif utile en conditions naturelles pour diminuer la turbulence et homogénéiser le milieu entourant les 2 soudures. Compte tenu de la méthode différentielle, cette perturbation peut être considérée comme négligeable.

La mise en place de la chambre se fait en 2 temps : le relèvement du levier 1 (fig. 2) fait descendre l'ensemble thermocouple, le relèvement du levier 2 fait ouvrir la pince, tout en maintenant l'ensemble thermocouple retiré. Une fois la feuille entre les mâchoires de la pince, les mouvements sont inversés automatiquement : la pince serre la feuille d'abord, puis l'ensemble thermocouple monte pour s'appuyer sur la feuille avec la pression réglée au préalable.

\section{Détermination de la pente à l'origine de la ciné- tique thermique}

Les déterminations de la cinétique peuvent se faire graphiquement à partir d'enregistrement sur papier ou de façon automatique par un dispositif électronique.

Dans le $1^{\text {er }}$ cas, la vitesse de variation de la température foliaire $(\Delta T / \Delta t)$ est enregistrée sur potentiomètre GRAPHISPOT à $\pm 0,08{ }^{\circ} \mathrm{C}$. La méthode de mesure manque cependant de précision : le temps de mesure étant très court (4 à $10 \mathrm{~s}$ ), une petite variation de la vitesse de déroulement du papier, l'imprécision personnelle introduite dans la détermination de la pente entraînent des erreurs qui ne sont pas négligeables.

Un dispositif électronique mesurant automatiquement le temps $\Delta$ t qui s'écoule pour que la feuille passe d'un seuil inférieur de température à un seuil supérieur préalablement choisis (fig. 1) a été mis au point par BERTOLINI (1977) et LEROUX (1978). Ce dispositif améliore la précision tout en augmentant la rapidité et la simplicité des mesures. 


\section{MISE EN OEUVRE DE LA MÉTHODE ET ÉTALONNAGE}

\section{A. Détermination de la partie "linéaire " de la cinétique thermique}

Sur une centaine de mesures enregistrées sur graphique, on a relevé les seuils minimaux et maximaux de température et la durée de la partie quasi linéaire de la cinétique thermique foliaire (fig. 1), en utilisant des feuilles de tournesol (Helianthus annuus L., var. 6501I.N.R.A.). Un début de mesure $2,5 \mathrm{~s}$ après l'allumage, une durée $\Delta \mathrm{t}$ de $7 \mathrm{~s}$, un seuil inférieur de $0,35^{\circ} \mathrm{C}$, un seuil supérieur de $1,75^{\circ} \mathrm{C}$ (soit une variation $\Delta \mathrm{T}$ de $1,40^{\circ} \mathrm{C}$ ) sont les valeurs qui ont conduit à la plus faible dispersion des mesures de Me aussi bien sur feuilles de tournesol que de maïs.

\section{B. Etalonnage en conditions de laboratoire}

La chambre présentée figure $4 a$ contenant un morceau de limbe d'environ $10 \mathrm{~cm}^{2}$ est placée sur une balance de précision. Toutes les 2 ou $3 \mathrm{mn}$, la masse fraîche du limbe et la cinétique thermique $\Delta T / \Delta t$ sont déterminées simultanément alors que la feuille se dessèche.

A partir des masses d'eau et de matière sèche surfaciques déterminées en fin d'expérience par séchage à l'étuve à $100{ }^{\circ} \mathrm{C}$, l'évolution de la masse d'eau surfacique $\mathrm{Me}$ au cours du dessèchement est établie de 2 façons :

$\mathrm{Me}_{\mathrm{g}}$ : par gravimétrie en déduisant à chaque instant de mesure la masse de matière sèche de la matière fraîche (la masse de matière sèche étant considérée comme constante pendant la durée des mesures),

$\mathrm{Me}_{\mathrm{m}}$ : par la méthode de la cinétique thermique en calculant le flux énergétique absorbé, $\Delta \phi a$, en fin d'expérience à partir de la relation ( $\left.5^{\prime}\right)$ après avoir déterminé MC par gravimétrie (équation 6). Les valeurs intermédiaires de MC se déduisent des cinétiques thermiques observées entre temps et permettent un calcul de $\mathrm{Me}_{\mathrm{m}}$.

Le décalage observé (fig. 5) entre les valeurs réelles, $\mathrm{Me}_{\mathrm{g}}$, et les valeurs mesurées par la méthode décrite, $\mathrm{Me}_{\mathrm{m}}$, pour des masses d'eau inférieures à 14$15 \mathrm{mg} . \mathrm{cm}^{-2}$ est dû au changement des propriétés optiques de la feuille, comme le montrent les travaux de SANTIBAÑEZ (1974). Ces valeurs, pour une feuille contenant $25 \mathrm{mg} . \mathrm{cm}^{-2}$ d'eau représentent un déficit de l'ordre de 40 à 45 p. 100 ; elles sont au-delà de la limite des variations écologiques de la masse d'eau.

En éliminant ces faibles valeurs, l'écart-type déterminé sur la base des déviations $\left(\mathrm{Me}_{\mathrm{g}}-\mathrm{Me}_{\mathrm{m}}\right)$ est de $\sigma=0,48$. On a donc 95 p. 100 de chance d'avoir une erreur inférieure à $1 \mathrm{mg} . \mathrm{cm}^{-2}$ dans l'estimation de la masse d'eau surfacique de la feuille. L'erreur moyenne est de $0,4 \mathrm{mg} . \mathrm{cm}^{-2}$. D'autre part, la pente de la droite de régression obtenue n'est pas significativement différente de 1 ce qui tend à valider les bases théoriques de la méthode.

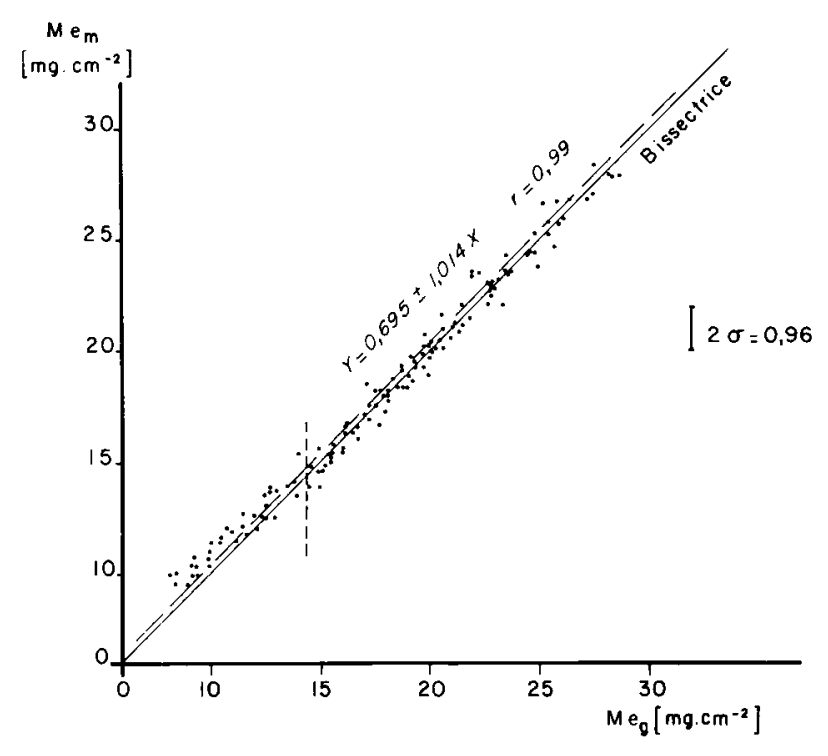

Figure 5

Correspondance entre les valeurs de masse d'eau surfacique déterminées simultanément par gravimétrie Me $\mathrm{g}_{\mathrm{g}}$ et par la méthode des cinétiques thermiques $\mathrm{Me}_{m}$ en conditions artificielles.

Relationship between values of water contents simultaneously determined by gravimetry $\mathrm{Me}_{g}$ and by the thermokinetics method $\mathrm{Me}_{m}$ in artificial conditions.

\section{Etalonnage en conditions naturelles}

La méthode d'étalonnage consiste à suivre le dessèchement d'un point sur une feuille par la méthode des cinétiques thermiques et à faire des prélèvements successifs de disques foliaires sur la même feuille, en des points analogues à celui de la mesure pour la détermination gravimétrique de la masse d'eau. Le nombre de prélèvements doit être assez limité ( 3 ou 4) pour ne pas altérer la masse d'eau dans le reste de la feuille. A la fin d'une période de dessèchement, la détermination de la cinétique thermique est suivie immédiatement d'un prélèvement de disques à l'endroit de la mesure, ce qui permet de connaître le contenu en eau final. A partir de ces valeurs finales, le contenu en eau initial est recalculé. Cette valeur est confrontée à la valeur mesurée initialement sur les disques prélevés sur l'autre côté de la feuille (fig. 6). Il existe un faible décalage des points obtenus par rapport à la première bissectrice. L'écart type obtenu en conditions naturelles est 1,9 fois plus grand que celui obtenu au laboratoire. L'erreur moyenne est de $0,72 \mathrm{mg} . \mathrm{cm}^{-2}$ ce qui est acceptable dans beaucoup de cas. Cet accroissement de l'erreur provient, entre autres, de la méthode d'étalonnage utilisée. Pour des feuilles de maïs, dont le contenu en eau surfacique est assez homogène, l'écart entre le contenu en eau de disques prélevés de part et d'autre de la nervure centrale est inférieur à 5 p. 100 de la masse d'eau totale. Pour des feuilles où la «symétrie " hydrique est moins bonne, un plus grand nombre de mesures pourra augmenter la précision.

\section{DISCUSSION ET CONCLUSION}

La méthode décrite permet d'étudier l'état hydrique de la feuille en conditions naturelles sans trop perturber ni sa physiologie, ni son environnement. Elle per- 


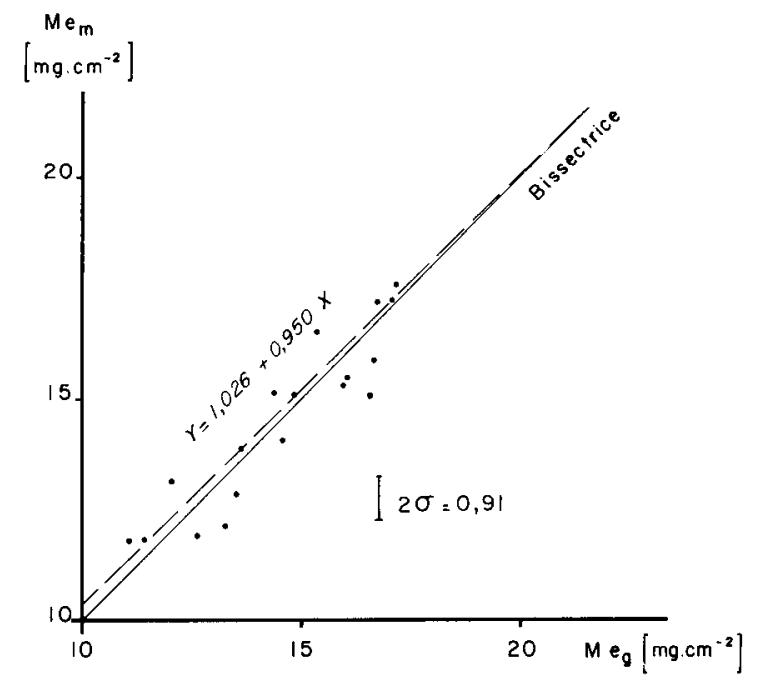

Figure 6

Correspondance entre les valeurs de masse d'eau surfacique obtenues par gravimétrie sur une moitié de la feuille $\mathrm{Me}_{\mathrm{g}}$ et celles déterminées par la méthode des cinétiques thermiques sur l'autre moitié de la feuille $\mathrm{Me}_{m}$ en conditions naturelles.

Relationship between water content values obtained by gravimetry on one side of the leaf $M e_{g}$ and those measured by the thermokinetics method on the other side of the leaf $\mathrm{Me}_{m}$ in natural conditions.

met donc de suivre les variations de la masse d'eau ou du déficit hydrique d'une même feuille au cours d'une longue période. C'est là un avantage essentiel par rapport à d'autres méthodes actuellement disponibles.

Sa précision est fonction de celle obtenue dans la détermination de la pente à l'origine de la cinétique thermique et de l'identité des conditions physiques et physiologiques des 2 points choisis sur la feuille (" effet de compensation parfait »).

Un écart de 2 à $3{ }^{\circ} \mathrm{C}$ suffit pour déterminer la pente à l'origine de la cinétique thermique. Cet écart est obtenu en $10 \mathrm{~s}$ environ en travaillant avec une intensité du flux incident de $210 \mathrm{~W} . \mathrm{m}^{-2}$. La comparaison de ces chiffres avec les valeurs normales des paramètres micrométéorologiques montre que l'altération du bilan énergétique introduite par la mesure reste dans la gamme normale de variation de ces paramètres. Dans les conditions naturelles, la feuille est soumise à des variations similaires (passage d'un nuage par exemple). Il faut d'ailleurs remarquer la courte durée de la mesure, ce qui permet de penser que son effet perturbateur est négligeable.

En conditions de laboratoire, la précision de la méthode pour la détermination de la teneur en eau est de l'ordre de $1 \mathrm{mg} . \mathrm{cm}^{-2}(5,6 \mathrm{p} .100)$ sur des feuilles de tournesol. L'erreur peut être attribuée principalement à l'hétérogénéité de la tache lumineuse.

En conditions naturelles, la valeur de l'erreur est environ 2 fois plus grande que celle obtenue au laboratoire. Malgré cela la précision de la méthode reste satisfaisante dans la majorité des cas. Il est fort probable que la principale cause d'imprécision en conditions naturelles avec le dispositif utilisé vienne du déplacement des thermocouples. De faibles déplacements dans la localisation des thermocouples ont pu se produire sous l'action du vent. Une meilleure homogénéisation de la tache lumineuse par utilisation d'un faisceau lumineux rigoureusement parallèle permettrait de remédier à ce défaut. Les fibres optiques ou le laser seraient à retenir.

D'autres améliorations techniques sont possibles :

- au niveau des capteurs : la mesure de la température de surface par radiométrie infrarouge différentielle constituera un important progrès dans l'avenir ;

- au niveau de l'automatisation : un système commandant simultanément l'allumage de la source d'énergie, l'enregistreur et l'obturateur, permettrait de laisser le système en place de façon à enregistrer jusqu'à une mesure toutes les 2 ou $3 \mathrm{mn}$ (temps minimal entre 2 mesures) pendant une longue période (jour et nuit) ; mais une fréquence aussi grande n'est certainement pas nécessaire dans la majorité des applications.

On peut aussi envisager la construction d'un dispositif plus simple et miniaturisé, facilitant l'utilisation au champ, qui se composerait d'un système d'éclairage à piles, d'un générateur d'électricité alimentant l'enregistreur et d'un jeu de pinces adaptées à plusieurs types de feuilles.

Reçu le 2 février 1983. Accepté le 6 février 1984.

\section{REMERCIEMENTS}

D. Leroux a réalisé le montage du dispositif électronique de mesure et D. Flura, le montage du dispositif d'apport d'énergie radiatif.

\section{RÉFÉRENCES BIBLIOGRAPHIQUES}

Bertolini J. M., 1977. Mesure de l'humidité surfacique des feuilles. Appareil dit de "Santibañez ». I.N.R.A., Station de Bioclimatologie, Versailles, 13 p., note interne.

Bielorai H., 1968. Beta ray gauging technique for measuring leaf water content changes of Citrus seedlings as affected by the moisture status in the soil. J. Exp. Bot., 19, 489-495.

Billings W. D., Morris R. J., 1951. Reflection of visible and infrared radiation from leaves of different ecological groups. Am. J. Bot., 38, 327-331.

Box J. E. Jr., Lemon E. R., 1958. Preliminary field investigations of electrical resistance-moisture stress relations in cotton and grain sorghum plants. Proc. Soil Sci. Soc. Am., 22, 193-196.
Buriol G. A., Menoux Y., Parcevaux S. de, 1984. Détermination de la masse d'eau et des propriétés optiques d'une feuille à partir de modifications de son bilan énergétique. II. Applications en conditions artificielles et naturelles. Agronomie, 4, (6), 501-506.

Buschbom U., 1970. Zur methodik kontinuierlicher WassergehaltBestimmungen and Blättern mittels $\beta$-Strahlenabsorption. Planta, 95, 146-166.

Durand R., 1970. Utilisation des thermocouples. In "Techniques d'étude des facteurs physiques de la biosphère ", M. Hallaire ed., I.N.R.A. Paris, 131-141.

Gardner W. R., Nieman R. H., 1964. Lower limit of water availability to plants. Science, 143, 1460-1462. 
Gezalyan M. G., 1968. Study of water content in leaves measured by dielectric permeability of water. Thèse, Akad. Nauk. Arm. SSR (en russe).

Gosse G., Parcevaux S. de, 1970. Application de l'absorption des ondes centimétriques à la mesure de l'humidité des végétaux, In "Techniques d'étude des facteurs physiques de la biosphère ", M. Hallaire ed., I.N.R.A. Paris, 359-369.

Hunt L. A., Impens I. I., 1968. Use of antitranspirants in studies of the external diffusion resistance of leaves. Oecol. Plant., 3, 1-6.

Impens I. I., 1966. Leaf wetness, diffusion resistances and transpiration rates of bean leaves (Phaseolus vulgaris L.) through comparison of « wet " and « dry » leaf temperature. Oecol. Plant., 1, 327-334.

Jarvis P. G., Slatyer R. O., 1966. Calibration of $\beta$-gauges for determining leaf water status. Science, 153, 78-79.

Jones H. G., 1973. Estimation of plant water status with the beta gauge. Agric. Meteorol., 11, 345-355.

Klemm W., 1959. Eine neue Methode zur Bestimmung der Holzfeuchterverlaufes in wachsenden Bäumen mit Hilfe von Gammastrahlen. Flora, 147, 465-470.

Knipling E. B., 1970. Physical and physiological basis for the reflectance of visible and near infrared radiation from vegetation. Remote Sensing Environ., 1, 155-159.

Kosmin P. L., Filipov P. L., 1968. Use of radar frequency in noncontact determination of leaf and stem moisture content. Soviet Plant Physiol., 15, 152-156.

Kreeb K., 1966. Die Registrierung des Wasserzustandes über die elektrische Leitfähigkeit der Blätter. Ber. Dtsch. Bot. Ges., 79, 150162.

Kreeb K., Abdelall R., 1973. Water relations, measured as electrical resistance of leaves, and correlation to growth and production, In R. O. Slatyer, «Réponse des plantes aux facteurs climatiques». UNESCO Paris, 497-503.

Kühn W., 1961. Zur Wassergehaltsbestimmung an lebenden Bäumen mittels schneller Neutronen und niederenergetischer Gammastrahlung. Kerntechnik, 3, 382-385.

Leroux D., 1978. Mise au point d'un dispositif électronique de mesure. Rapport de stage, I.N.R.A., Bioclimatologie, Versailles, 22 p.

Linacre E. T., 1972. Leaf temperatures, diffusion resistances and transpiration. Agric. Meteorol., 10, 365-382.

Mederski H. J., 1961. Determination of internal water status of plants by beta ray gauging. Soil Sci., 92, 143-146.

Mederski H. J., Alles W., 1968. Beta gauging leaf water status : influence of changing leaf characteristics. Plant Physiol., 43, 470-472.

Nakayama F. S., Ehrler W. L., 1964. Beta ray gauging technique for measuring leaf water content changes and moisture status of plants. Plant Physiol., 39, 95-98.
Namken L. N., Lemon E. R., 1960. Field studies of internal moisture relations of the corn plant. Agron. J., 53, 643-646.

Parcevaux S. de, 1972a. Contribution à l'étude des échanges gazeux au niveau des feuilles. Thèse Dr. d'Etat, Faculté d'Orsay - Paris Sud, 86 p. + annexes.

Parcevaux S. de, 1972 $b$. Aspects biophysiques des échanges entre les feuilles et le milieu environnant. Oecol. Plant., 7, 371-401.

Parcevaux S. de, Perrier A., 1973. Bilan énergétique de la feuille. Application de l'étude des cinétiques de température à la détermination des résistances aux flux gazeux. In $R$. $O$. Slatyer : "Réponse des plantes aux facteurs climatiques». UNESCO, Paris, 127-135.

Perrier A., 1970. Mesure des températures de surface par radiométrie infrarouge. In «Techniques d'étude des facteurs physiques de la biosphère ", M. Hallaire ed., I.N.R.A., Paris, 169-178.

Perrier A., 1971, Leaf temperature measurements. In Z. Sestak, J. Catsky, P. G. Jarvis: « Plant photosynthetic production, Manual of methods ». W. Junk, The Hague, 632-671.

Perrier A., Itier B., 1975. Valeurs des températures de surface du milieu naturel et leur détermination en fonction des capteurs. In «La mesure des températures de surface». Soc. Fr. des thermiciens, Symp., La Baule, 5-7 mai 1975, 125-135.

Santibañez F., 1974. Etude des variations de la teneur en eau des feuilles au moyen d'une méthode rapide et non destructive. Thèse Université Paris VII, $78 \mathrm{p}$

Schulze E. D., 1970. Der $\mathrm{CO}_{2}$ Gaswechsel der Buche (Fagus silvatica L.) in Abhängigkeit von den Klimafaktoren im Freiland. Flora, $159,177-232$.

Sinclair T., Hoffer R. M., Schreiber M., 1971. Reflectance and internal structure of leaves from several crops during a growing season. Agron. J., 63, 864-868.

Siri W. E., 1949. Isotopic tracers and nuclear radiations. McGraw Hill, N.Y., 58 p.

Thomas J. R., Namken L. N., Oerther G. F., Brown R. G., 1971. Estimating leaf water content by reflectance measurements. Agron. $J ., 63,845-847$.

Unger K., 1963. Experimentelle Untersuchungen mit Hilfe von ionisierenden Strahlen und Neutronen zur Bestimmung der Witterungsbedingten Wachtumsintensität von Kulturpflanzen am natürlichen Standort. Veröffentlichungen Int. Agrometeorol, Karl Marx Univ. II, 2, $135 \mathrm{p}$.

Unger K., 1968. Methodische Betrachtungen zur modellmäbigen Darstellung des Wasserhaushaltes von Kulturpflanzen. In «Biophysik pflanzlicher Systeme, Quedlinburg », Studia Biophysica, 11, 7987.

Yamada Y., Tamai S., Myaguchi T., 1961. The measurement of thickness of leaves using ${ }^{35} \mathrm{~S}$. Proc. 2nd Japan Conf. on Radioisotopes, 1958 (Us-AEC-Tr-4482), 1692-1700. 